\title{
Understanding Factors Associated With Children's Motivation To Engage In Recess-Time Physical Activity
}

Merav W. Efrat, California State University, Northridge, USA

\begin{abstract}
Physical activity is linked with health and academic benefits. While recess provides the greatest opportunity for children to accumulate physical activity, most children are not motivated to engage in sufficient amounts of physical activity during recess. Research demonstrates a strong relationship between self-efficacy and children's motivation to engage in physical activity. The purpose of this study was to explore whether in the context of recess there is a relationship between physical activity self-efficacy and children's motivation to engage in physical activity. One hundred and sixty-one students were recruited from three elementary schools. Physical activity self-efficacy (barrier and task) and physical activity data were collected utilizing a self-efficacy instrument and accelerometers. This study found evidence of two types of barrier self-efficacy, adult encouragement barrier self-efficacy and other barrier self-efficacy, neither of these types of barrier self-efficacy, nor task self-efficacy were found to be significantly related to motivation to engage in physical activity during recess. Given research suggesting that other types of physical activity self-efficacy may be useful in understanding children motivation to engage in physical activity, more research in this area is needed. Future research may consider exploring the relationship between asking efficacy, environmental change efficacy and motivation to engage in recess-time physical activity. This research can assist practitioners and researchers identify modifiable correlates associated with children's recess time physical activity. Such evidence could be utilized to develop interventions that are effective at increasing the amount of physical activity children accumulate during recess time.
\end{abstract}

Keywords: Children; Recess; Physical Activity; Motivation

\section{INTRODUCTION}

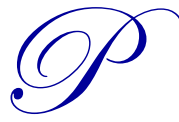

hysical activity is linked with lower rates of obesity as well as a substantial number of health and academic benefits (Strong et al. 2005; Buck Hillman, Castelli, 2008; Castelli Hillman, Buck \& Erwin, 2007). Inactive elementary school age children, compared to their more active peers, have poorer cardiovascular risk profiles, bone health and mental health as well as poorer performance on a variety of cognitive tasks, and academic assessments (Strong et al. 2005; Buck Hillman, Castelli, 2008; Castelli Hillman, Buck \& Erwin, 2007). In spite of substantial health and academic benefits associated with physical activity, many environmental and social barriers prevent elementary school-age children from meeting the current physical activity recommendations of 30 to 60 minutes of moderate to vigorous physical activity (MVPA) on a daily basis (Troiano, 2007). Social and environmental barriers often preventing children from engaging in physical activity during other segments of the day (active travel to school, physical education classes, and extracurricular activities) do not pertain to unstructured in-school free time (i.e., recess). For instance, while distance and traffic concerns prevent many children from actively commuting to school, the playground is easily accessible and devoid of traffic dangers. While lack of transportation prevents many children from engaging in afterschool extracurricular physical activity, children do not need transportation to access the playground during recess time. Lastly, in the United States, elementary school-aged children receive on average 125 minutes per week of scheduled recess (National Center for Education statistics, 2006). Indeed, elementary school-age children accumulate more physical activity in unstructured, rather than structured environments (Pate et al., 1996). 
Despite its potential, many children lack the motivation necessary to fully maximize recess to reach current physical activity benchmarks (Ridgers, Stratton, \& Fairclough, 2005; Stratton, 1999, 2000). Motivation is inferred through the presence of three behavioral indicators: choice, effort, and persistence (Weiner, 1990). Choice is when an individual who is given alternatives voluntarily chooses to engage in a particular behavior. Effort entails the amount of cognitive or physical effort an individual invests when accomplishing a particular task. Persistence relates to the time an individual spends on a particular task. Accordingly, children who are motivated to engage in physical activity during recess are likely to voluntarily choose to engage in greater amounts of MVPA during recess than their less motivated peers. Data indicates that boys spend only $32.9 \%$, and girls only $25.3 \%$, of their recess time engaged in MVPA (Ridgers, Stratton, \& Fairclough, 2005).

In the context of recess-time physical activity, choice to engage in physical activity relates to a child voluntarily choosing to engage in physical activity. For example, when a child chooses to play handball during recess, he or she is demonstrating free choice to engage in physical activity. On the other hand, participation in a mandatory physical education class would not be an example of free choice to engage in physical activity. Effort relates to the amount of physical energy an individual expends during physical activity. Physical activity intensity or effort is frequently classified in three distinct categories (U.S. Department of Health and Human Services, 1996). Those categories are: light-intensity physical activity (LPA), moderate-intensity physical activity (MPA) or vigorous-intensity physical activity (VPA). LPA refers to light activities that require very little physical effort, such as walking slowly. MPA refers to any activity which results in the increase of an individual's breathing or heart rate. VPA refers to any activity that results in large increases in breathing or heart rate during which conversation is difficult ("Physical activity for everyone", n.d). Lastly, physical activity persistence relates to how long someone spends engaging in physical activity.

Elementary school-aged children have problems with choice, effort, and persistence that prevent them from engaging in sufficient amounts of physical activity during recess time. Many elementary school-aged children do not choose to engage in physical activity during their recess time (Ridgers, Stratton, \& Fairclough, 2005; Stratton, 1999, 2000). Zask, Van Beurden, Barrnett, Brooks and Dietrich (2001) observed that during recess time approximately $50 \%$ of elementary school-aged boys, compared to $26.5 \%$ of elementary school-aged girls, chose to engage in physical activity during recess. In addition to problems with choice, data suggests that elementary schoolaged children do not expend sufficient effort when they engage recess time physical activity. For example, Stratton $(1999,2000)$ found that children spent only $15 \%$ to $40 \%$ of their recess time engaged in MVPA. Elementary schoolaged children also have problems with persisting when engaged in recess time physical activity. McKenzie et al. (1997) found that most elementary school-aged children only spent the first three minutes of their recess time engaged in MVPA.

Given the potential for children to accumulate physical activity during recess, increasing motivation to engage in recess time physical activity is a promising approach to enabling more children to reach physical activity benchmarks. One way to increase children's motivation to engage in recess time physical activity is to design interventions that incorporate modifiable correlates of children's physical activity. While many modifiable correlates of children's physical activity have been identified, research indicates that there is a strong relationship between barrier and task physical activity self-efficacy and children's choice, effort, and persistence to engage in physical activity (Annesi, 2006; Annesi et al., 2007; Chase, 2001; Foley et. al., 2008, Norton, 2003; Sharma, Wagner., \& Wilkerson, 2005; Trost et al., 1999a; Trost, Pate, Ward, Saunders, \& Riner,1999b ). Self-efficacy "is a judgment of one's capability to accomplish a certain level of performance." (Bandura, 1986, p. 391). Accomplishing a certain level of performance requires both confidence in one's capabilities (task self-efficacy), as well as confidence in one's abilities to overcome potential barriers to execute the particular action (barrier selfefficacy) (Glanz, Rimer, \& Lewis, 2002). Limited evidence suggests that psychosocial correlates of children's physical activity, such as self-efficacy, can vary during different segments of the day (Ommundsen et al. (2006). To date no research has explored whether in the context of recess there is a relationship between self-efficacy and children's motivation to engage in physical activity. The purpose of this study was to determine whether in the context of recess there is a relationship between children's physical activity self-efficacy and children's motivation to engage in physical activity during recess (percent of recess time child spent engaged in MVPA). 


\section{METHODS}

A total of 161 (67\% of the sampling frame) ethnically and socioeconomically diverse students recruited from three elementary schools participated in this study. Initially 165 students were recruited. However, three students returned their consent forms after data collection was already completed and one was absent during the data collection period. Participants' demographics are found in table 1.

Table 1. Participants Demographics

\begin{tabular}{l|c}
\hline & Total Number of Participants: 161 \\
\hline Gender & $69(42.9 \%)$ \\
\hline Male & $92(57.1 \%)$ \\
\hline Female & $45(28.0 \%)$ \\
\hline Ethnicity & $77(47.8 \%)$ \\
\hline White & $18(11.2 \%)$ \\
\hline Latino & $13(8.1 \%)$ \\
\hline African American & $3(1.9 \%)$ \\
\hline Asian & $2(1.2 \%)$ \\
Filipino or Pacific Islander & $3(1.9 \%)$ \\
\hline American Indian or Alaska Native & \\
\hline Other & \\
\hline
\end{tabular}

\section{Measures}

\section{Recess Time Physical Activity Self-Efficacy Instrument}

In this study self-efficacy was measured using the Recess Physical Activity Self-Efficacy Scale. Items 1 to 11 assessed barrier self-efficacy and were adapted from the Exercise Barriers Self-Efficacy Scale for Children developed by Annesi et al. (2005) to assess 7-to-12-year-old children's ability to overcome personal, social, and environmental barriers associated with engaging in physical activity. Cronbach's alpha for Annesi's instrument were 0.67 and 0.70 in girls ages 9 to 10 and 0.78 and 0.85 for boys 9 to 10 (Annesi et al., 2005). In this study the modified barrier self-efficacy items had a coefficient alpha of 0.78 . Modified items began with the stem, "I am confident I can exercise during recess even if." Further, an original item stating, "I am sure I can exercise even if an instructor did not offer me any encouragement" was slightly modified. The word "instructor" was substituted with references to other adults in the school context that potentially can encourage a child to engage in physical activity (i.e., teacher, yard aid, and coach). The instrument used a 5-point Likert scale ranging from 1 (not at all confident) to 5 (definitely confident).

The last three items (items 12 to 14) on the self-efficacy instrument for this study assessed task self-efficacy. These items were adapted from a task self-efficacy instrument developed by Foley et al. (2008) to assess 11-to-13 year-old children's physical activity self-efficacy. Foley et al.'s instrument which was piloted before use was reliable $(\alpha$ $=0.95$ ) and assessed children's confidence engaging in various levels of physical activity intensity (i.e., light, moderate, vigorous) over a progressively longer period of time (i.e., 10, 20, and 30 minutes). Since this study focused on physical activity during recess, which is only 20 minutes in duration, items were slightly modified to assess children's confidence to engage in various levels of physical activity intensity during the entire duration of recess. Specifically, three single item measures were used to assess each of the following types of task self-efficacy: 1) light physical activity task self-efficacy; 2) moderate physical activity task self-efficacy; and 3) vigorous physical activity self-efficacy. The same 5-point Likert scale described previously to assess barrier self-efficacy was utilized to assess each of the three task self-efficacy items.

\section{Motivation Data}

In this study the children's motivation to engage in physical activity during recess was measured using the unidirectional Actigraph AM7164 accelerometers which are a valid instrument for discriminating between sedentary, light, moderate, and vigorous physical activity in children (Puyau, Adolph, Vohra, \& Butte, 2002). The physical activity motivation data described below is the pre-intervention baseline data collected for an intervention study 
which has already been published (Efrat, 2013). Prior to the start of this study, the Active Living Foundation, who loaned the investigator the accelerometers, calibrated the accelerometers using a standard protocol to ensure reliability of measurement. To determine the amount of MVPA participants' accumulated during recess, the investigator utilized the accelerometers' default activity counts, which are similar to those used by Trost, Pate, Sallis, et al. (2002) and set the monitor to a 15 second sampling interval or epoch. The recorded activity counts for each 15 second epoch were downloaded to a personal computer. Utilizing the Actilife 4.2.0 software, the 15 second movement counts were re-integrated into one minute movement counts and converted into an Excel activity output file. Although all three of the schools participating in this study formally provided their students with the opportunity to engage in a 20-minute morning recess, classroom teachers occasionally release their students to recess a bit later than the formal recess start time. To account for these variations, the investigator and research assistants documented, on all data collection days, the exact time students in each participating class were released to recess along with exact end time of recess. Utilizing this information, the investigator was able to calculate the percent of available recess time each participant spent in MVPA. This variable was utilized for all later data analysis relating to the physical activity variable.

\section{Procedures}

Before collecting data, the researcher obtained Institutional Review Board approval from the Los Angeles Unified School District Committee for External Research Review and the University of Southern California Office for the Protection of Research Subjects. Both a signed parental consent form and a child assent form were required for participation in the study. Before administering the recess time physical activity self-efficacy instrument, the researcher and her research assistants implemented several brief activities. The purposes of these activities were to increase participants' understanding of survey items relating to self-efficacy. Research findings indicate that providing children with concrete examples of physical activities and sedentary activities increases children's understanding of the definition of physical activity (Trost et al., 2000). Additionally, Bandura (2006) suggests that providing children with concrete examples of the scale that will be used to assess self-efficacy may decrease children's misconceptions relating to values used on a self-efficacy scale.

To increase participants' understanding of the concept of physical activity, the researcher provided a verbal description of physical activity, various physical activities children can engage in during recess, as well as sedentary activities children commonly engage in during recess. To increase participants' understanding of the concept of light, moderate, and vigorous physical activity, the researcher read participants a definition of each physical activity intensity level and encouraged them to engage in a brief bout of activity at that particular intensity. For instance, after reading the definition of light intensity physical activity, the researcher had participants walk at a slow pace. After reading the definition of moderate intensity physical activity, the researcher had participants walk at a brisk pace. Lastly, after reading a definition of vigorous physical activity, the researcher had participants sprint a short distance.

Additionally, the investigator and her research assistants marked the playground area with 5 chalk markings which were progressively farther in distance from each other. Subsequently participants were asked to rate their level of confidence as it relates to their ability to jump to each of the distances. They used the same scale descriptors used on the physical activity self-efficacy instrument to rate their level of confidence (e.g., not at all confident, somewhat confident, definitely confident). After assessing their level of confidence, the participants attempted to jump the specific distance. After participants jumped, the investigator and her research assistants ensured that participants understood the scale descriptors that would be utilized to assess their level of confidence on the self-efficacy instrument. They did so by explaining the difference between the levels of confidence when clarification was necessary (e.g., defining definitely confident vs. somewhat confident). Following these activities, the investigator and her research assistants implemented the recess time self-efficacy physical activity instrument. To maximize children's understanding of each item, the investigator read each question aloud to study participants.

Due to the limited number of accelerometers (i.e., 30 monitors), it typically took the investigator and research assistants three days to collect data from the participants in each of the three schools. (i.e., two days to gather data from most participants and one reserved for make ups). Make-ups were needed when participants were absent, monitors malfunctioned, and weather prevented students from engaging in their regularly scheduled recess. Before 
attaching accelerometers on study participants, the investigator described to the students the function of the accelerometer. To prevent the participants from intentionally increasing their activity levels when wearing an accelerometer, they were informed that the investigator was writing a non-fiction book about children's physical activity during recess. Further, participants were told that to ensure that this book provided adults with valuable information on ways to keep children healthy, it was critical that participants engage in their normal recess time activities. Lastly, participants were informed that should their accelerometer become loose during recess they should seek out the investigator or her research assistants who would properly re-attach the monitors.

Following accelerometer instruction, the investigator and her research assistants placed the accelerometers on the participants' right hip by clipping it onto their waist using the elastic belt that comes with the accelerometer. Research suggests that in free living conditions, such as during recess, placing the accelerometer on the hip may be most accurate (Heil, 2006). At the end of the data collection day, participants took off their accelerometer and the investigator downloaded the physical activity data to a computer.

\section{Data Analysis}

All data were analyzed using SPSS for Windows (17.0). Before data was analyzed, the researcher ran an exploratory factor analysis to determine the number of constructs the self-efficacy instrument was measuring as well as a Cronbach's alpha test to determine the instrument's reliability. Descriptive statistics for self-efficacy variables and physical activity were computed. A Spearman's rank correlation was run to determine whether there was a relationship between self-efficacy and children's motivation to engage in physical activity during recess.

\section{RESULTS}

The barrier self-efficacy scale used in this study differed from prior physical activity barrier self-efficacy scales in that it included three items, rather than one, that specifically assessed a child's ability to be less dependent on an adult's encouragement to prompt them to be active during recess. A principal component analysis with varimax rotation was conducted to ascertain whether the three items relating to adult encouragement might measure a construct that differs from the rest of the barrier self-efficacy items. Analysis found that the barrier self-efficacy scale did consist of two types of barrier self- efficacy. Analysis identified one construct consisting of items 1- 8 with unique factor loadings greater than 0.32 and another construct consisting of items 9 - 11 also with unique factor loadings greater than .70 . Close examination of the items falling into these two different constructs suggest that one construct was measuring a type of barrier self-efficacy that is associated with a child's ability to be less dependent on an adult's encouragement to prompt them to be active in unstructured environments such as recess. For instance, items 9,10 , and 11 related to a child's confidence in their ability to engage in physical activity during recess even if they did not receive encouragement from an adult in the school context, such as a teacher, a coach, or a yard aid. In contrast, the other construct was measuring a child's confidence in their ability to overcome other common personal, social, and environmental physical activity barriers. For instance, items $1-8$, were related to other common barriers preventing children from engaging in physical activity such as: boredom, having to exercise alone, lack of fun, and disliking of a particular activity. Therefore, subsequent analysis utilizing the barrier self-efficacy variable examined adult encouragement barrier self-efficacy and other barrier self-efficacy. Reliability Cronbach's alpha for, adult encouragement barrier self-efficacy and other barrier self-efficacy were $0.70,0.74$, respectively.

Descriptive statistics for all participants were computed. Table 2 provides the descriptive statistics for other barrier self-efficacy, adult encouragement barrier self-efficacy, light physical activity task self-efficacy, moderate physical activity task self-efficacy, vigorous physical activity task self-efficacy and motivation to engage in physical activity for all study participants. 
Table 2. Descriptive statistics for Self-Efficacy and Motivation to Engage in Physical Activity

\begin{tabular}{|c|c|c|c|}
\hline & $N$ & Mean & Std. Deviation \\
\hline Other Barrier Self-Efficacy & 158 & 3.43 & 0.86 \\
\hline Adult Encouragement Barrier Self-Efficacy & 158 & 4.17 & 0.97 \\
\hline Light Physical Activity Task Self-Efficacy & 158 & 4.14 & 1.19 \\
\hline Moderate Physical Activity Task Self-Efficacy & 158 & 4.01 & 0.96 \\
\hline Vigorous Physical Activity Task Self-Efficacy & 156 & 3.75 & 1.25 \\
\hline Motivation to Engage in Physical-Activity & 158 & $34 \%$ & 0.24 \\
\hline
\end{tabular}

A Spearman's rank correlation analysis was run to determine whether self-efficacy was positively related to motivation to engage in physical activity during recess. As presented in Table 3 , results indicated that adult encouragement barrier self-efficacy, other barrier self-efficacy, light physical activity task self-efficacy, moderate physical activity task self-efficacy, and vigorous physical activity task self-efficacy were not significantly related to effort. These findings were unexpected.

Table 3. Correlation Coefficients for the Association between Self-Efficacy, and Motivation to Engage in Physical Activity during Recess

\begin{tabular}{l|c}
\hline & Motivation to Engage in Physical Activity during Recess \\
\hline Other Barrier Self-Efficacy & .14 \\
\hline Adult Encouragement Barrier Self-Efficacy & .08 \\
\hline Light Physical Activity Task Self-Efficacy & -.07 \\
\hline Moderate Physical Activity Task Self-Efficacy & .02 \\
\hline Vigorous Physical Activity Task Self-Efficacy & .15 \\
\hline Other Barrier Self-Efficacy & 1 \\
\hline
\end{tabular}

\section{DISCUSSION}

It was expected that there would be a positive relationship between self-efficacy and motivation to engage in physical activity in the context of recess. This did not occur. While this study found evidence of two types of barrier self-efficacy, adult encouragement barrier self-efficacy and other barrier self-efficacy, neither of these types of barrier self-efficacy, nor light physical activity task self-efficacy, moderate physical activity task self-efficacy or vigorous physical activity task self-efficacy were found to be significantly related to motivation to engage in physical activity during recess. These findings are in line with the limited research suggesting that psychosocial correlates of physical activity can vary during different physical activity contexts (Ommundsen et al., 2006). Research that has found an association between self-efficacy and choice to engage in physical activity primarily examined physical activity in the context of unstructured afterschool physical activity and used self report physical activity data which is unreliable in children under the age of 10 (Trost, Morgan, Saunders, Ward, \& Pate, 2000). While the studies linking self-efficacy to effort and persistence utilized more reliable assessments of physical activity (accelerometers), they considered physical activity as uni-dimensional construct which did not account for potential context or location specific variations on children's physical activity (i.e., structured vs. unstructured, inschool vs. after school) (Foley et al., 2008; Trost et al.,1999b, 1999b; Zakarian et al., 1994).

The findings from this study suggest that in the context of recess, self-efficacy and motivation to engage in physical activity are unrelated. There are limitations to this study that may also explain these results including: the type of self-efficacy data that was collected and the duration of recess time physical activity data that was collected. Most studies suggest that the construct of physical activity self-efficacy consists of two types of self-efficacy (task selfefficacy and barrier self-efficacy). A limited number of studies have found evidence of other types of physical activity self-efficacy that may be useful in understanding children's motivation to engage in physical activity. For instance, in the context of unstructured afterschool physical activity, Ryans and Dzewaltowski (2002) provide evidence that in addition to barrier self-efficacy, two other types of self-efficacy maybe useful in understanding how children can successfully overcome various barrier that are associated with engaging in afterschool unstructured physical activity. Those types of efficacy are: environmental change efficacy, which relates to a child's ability to find and create environments that support physical activity, as well as asking efficacy which relates to a child's ability to ask other to be active with him or her. The physical activity environment during recess is quite similar to the afterschool unstructured physical activity environment in that it typically does not entail an adult actively 
initiating and maintaining physical activity. In structured physical activity environments, such is physical education instruction, children do not need to initiate physical activity (Efrat, 2013). Instead, an adult chooses the activity in which the child will engage, and puts forth the effort to locate necessary equipment and an appropriate location to play the game (Efrat, 2013). In contrast, in order for a child to accumulate moderate to vigorous physical activity during unstructured physical activity environments, a child must be able to ask their peers to be active with him or her (asking self-efficacy), and make the effort to get any necessary equipment or locate an appropriate location to engage in the particular game (environmental change efficacy). Future research may consider exploring the relationship between asking self-efficacy, environmental change efficacy and motivation to engage in physical activity during recess.

This study's results may also be explained by the limited number of days of recess time physical activity data collected (only one day of morning-time recess). Currently research has not determined whether monitoring children's recess time physical activity during one recess period is representative of the amount of physical activity they typically accumulated during recess.

\section{CONCLUSION}

This study explored whether in the context of recess there is a relationship between physical activity self-efficacy (barrier and task) and children's motivation to engage in physical activity. While this study found evidence of two types of barrier self-efficacy, adult encouragement barrier self-efficacy and other barrier self-efficacy, neither of these types of barrier self-efficacy, nor light physical activity task self-efficacy, moderate physical activity task selfefficacy or vigorous physical activity task self-efficacy were found to be significantly related to motivation to engage in physical activity during recess. Given research suggesting that other types of physical activity selfefficacy may be useful in understanding children motivation to engage in physical activity, more research in this area is needed. This research can assist practitioners and researchers identify modifiable correlates associated with children's recess time physical activity. Such evidence could be utilized to develop interventions that are effective at increasing the amount of physical activity children accumulate during recess time.

\section{ACKNOWLEDGEMENTS}

The author would like to thank the principals, teachers, and children at the schools that participated in the study. The author would also like to thank Anya Hobley and Keylaundra McClelland who helped with the data collection. In addition, the author would like to thank Younjoo Oh for his assistance with data analysis, John Schneider for his technical assistance with utilizing the Actigraph monitors and the Active Living Research Program of the Robert Wood Johnson Foundation, the National Center for Health Statistics and the National Cancer Institute for the loan of the accelerometer equipment used for this study.

\section{AUTHOR BIOGRAPHY}

Dr. Merav Efrat is an Assistant Professor in the Department of Health Sciences at Cal State University Northridge. She has over ten years of professional work experience as a Health Educator and International Board Certified Lactation Consultant. Her research focuses on developing and evaluating interventions that reduce childhood obesity, particularly among disadvantaged communities. Specifically, in an attempt to reduce the prevalence of childhood obesity, she is interested in examining the efficacy of interventions that can be used to promote physical activity among elementary school-age children, as well as to promote breastfeeding rates. 


\section{REFERENCES}

Annesi, J. J. (2006). Relations of physical self-concept and self-efficacy with frequency of voluntary physical activity in preadolescents: Implications for after-school care programming [Electronic version]. Journal of Psychosomatic Research, $61(4), 515-520$.

Annesi, J. J., Faignenbaum, A. D., Wesscott, W. L., Smith, A. E., Unruh, J. L., \& Hamilton, F. G. (2007). Effects of the youth fit for life protocol on physiological mood, self-appraisal, and voluntary physical activity changes in African American preadolescents: Contrasting after-school care [Electronic version]. International Journal of Clinical and Health Psychology, 7(3), 641-659.

Annesi, J. J., Westcott, W. L., Faigenbaum, A. D., \& Unruh, J. L. (2005). Effects of a 12-week physical activity protocol delivered by YMCA after-school counselors (youth fit for life) on fitness and self-efficacy changes in 5-12 year old boys and girls [Electronic version]. Research Quarterly for Exercise and Sport, 76(4), 468-469.

Bandura, A. (1986). Social foundations of thought and action: A social cognitive theory. Englewood Cliffs, NJ: Prentice-Hall, Inc.

Bandura, A. (2006). Guide for constructing self-efficacy scales. In F. Pajares \& T. Urdan (Eds.). Self-efficacy beliefs of adolescents (pp. 307-337). Charlotte, NC: Information Age Publishing.

Buck, S. M. (2008). Aerobic fitness and attentional control in preadolescent children. (Doctoral dissertation, University of Illinois at Urbana-Champaign, 2008). Dissertation Abstracts International, 68(11-B), 7138.

Buck, S. M., Hillman, C. H., \& Castelli, D. M. (2008). The relation of aerobic fitness to stroop task performance in preadolescent children. [Electronic version]. Medicine and Science in Sports and Exercise, 40(1)166-172.

Castelli, D. M., Hillman, C. H., Buck, S. M., \& Erwin, H. E. (2007). Physical fitness and academic achievement in third-and fifth-grade students [Electronic version]. Journal of Sport \& Exercise Psychology, 29, 239-252.

Chase, M.A. (2001). Children's self-efficacy: Motivational intentions and attributions in physical education and sports [Electronic version]. Research Quarterly for Exercise and Sport, 72(1), 47-54.

Efrat, M.W. (2013). Exploring effective strategies for increasing the amount of moderate-to-vigorous physical activity children accumulate during recess: a quasi-experimental intervention study. Journal of School health, 83(4), 265-272).

Foley, L., Prapavessis, H., Maddison, R., Burke, S., McGowan, E., \& Gillanders, L. (2008). Predicting physical activity intention and behavior in school-age children [Electronic version]. Pediatric Exercise Science, 20(3), 342-356.

Glanz, K. Rimer, B. K., \& Lewis, F. M. (Eds.). (2002). Health Behavior and Health Education. San Francisco: Jossey-Bass.

Heil, D. P. (2006). Predicting activity energy expenditure using the actical activity monitor [Electronic version]. Research Quarterly for Exercise and Sport, 77(1), 64-80.

Mulvihill, C., Rivers, K., Aggleton, P. (2000) A qualitative study investigating the views of primary-age children and parents on physical activity [Electronic version]. The Health Education Journal, 59(2), 166-179.

National Center for Education Statistics (2006). Calories in, calories out: Food and exercise in public elementary schools, 2005 Retrieved February 11, 2010, from http://nces.ed.gov/pubs2006/nutrition/index.asp

Norton, D. E. (2003). Eating and exercise behaviors in preadolescents: Parental influence? Unpublished Ph.D., University of California, San Francisco.

Ommendsen, Y., Klasson-Heggebo, L., Anderssen, S.A. (2006). Psycho-social and environmental correlates of location-specific physical activity among 9- and 15- year old Norwegian boys and girls: European Youth Heart Study [Electronic version]. International Journal of Behavioral Nutrition and Physical Activity, 3(3).

Pate R. R., Baranowski T., Dowda M., \& Trost S. G. (1996). Tracking of physical activity in young children [Electronic version]. Medicine Science in Sports and Exercise, 28, 92-6.

Puyau, M. R., Adolph, A. L., Vohra, F. A., \& Butte, N. F., (2002) validation and calibration of physical activity monitors in children [Electronic version]. Obesity Research, 10(3), 150-157.

Ridgers, N. D., Stratton, G., \& Fairclough, S. J. (2005). Assessing physical activity during recess using accelerometry [Electronic version]. Preventive Medicine, 41,102-107.

Ridgers, N.D., Stratton, G., \& Fairclough, S. J. (2006). Physical activity levels of children during school playtime [Electronic version]. Sports Medicine, 36(4), 359-371.

Robert Wood Johnson Foundation (2007). Recess rules: Why the undervalued playtime may be America's best investment for healthy kids and healthy schools.

Sharma, M., Wagner, D. I., \& Wilkerson, J. (2005). Predicting childhood obesity prevention behaviors using social cognitive theory [Electronic version]. International Quarterly of Community Health Education, 24(3), 191-203.

Stratton G. (1999). A preliminary study of children's physical activity in one urban primary school playground: differences by sex and season [Electronic version]. Journal of Sport Pedagogy, 5, 71-81.

Stratton G. (2000). Promoting children's physical activity in primary school: An intervention study using playground markings [Electronic version]. Ergonomics, 43(10), 1538-1546.

Strong, W. B., Malina, R. M., Blimkie, C. J. R., Daniels, S. R., Dishman, R. K., Gutin, B. G., et al. (2005). Evidence based physical activity for school age youth [Electronic version]. Journal of Pediatrics, 146, 732-737.

Troiano, R., P., Berrigan, D., Dood, K. W., Masse, L. C, Tilert, T., \& McDowell, M. (2007). Physical Activity in the United States measured by accelerometer [Electronic version]. Medicine \& Science in Sports \& Exercise, 40(1), 181-188 
Trost, S.G., Morgan, A. M., Saunders, G. F., Ward, D. S., Pate, R. R. (2000). Children's understanding of the concept of physical activity [Electronic version]. Pediatric Exercise Science 12, 293-299.

Trost S. G, Pate R R., Sallis J. F., Freedson, P. S., Taylor, W. C., Dowda, et al. (2002). Age and gender differences in objectively measured physical activity in youth. [Electronic version]. Medicine and Science in Sports and Exercise, 34, 350-355.

Trost, S.G., Pate, R. R., Ward, D. S., Saunders, R., \& Riner, W. (1999a). Correlates of objectively measured physical activity in preadolscent youth [Electronic version]. American Journal Preventive Medicine, 17(2), 120- 126.

Trost, S. G., Pate, R.R., Ward, D.S., Saunders, R., \& Riner, W. (1999b) Determinants of physical activity in active and lowactive, sixth grade African American youth [Electronic version]. Journal of School Health, 69(1), 29-34.

Weiner, B. (1990). History of motivational research in education. Journal of Educational Psychology, 82(4), 616-622.

Zakarian, J. M., Hovell, M. F., Hofstetter, C. R., Sallis, J. K., \& Keating K. J. (1994). Correlates of vigorous exercise in predominantly low SES and minority high school population [Electronic version]. Preventive Medicine, 23, 314-321. 
NOTES 